\title{
Burden of Migraine in Japan: Results of the ObserVational Survey of the Epidemiology, tReatment, and Care Of MigrainE (OVERCOME [Japan]) Study
}

\author{
Yasuhiko Matsumori · Kaname Ueda (D) - Mika Komori · \\ Anthony J. Zagar • Yongin Kim • Dena H. Jaffe (D) · Takao Takeshima • \\ Koichi Hirata
}

Received: October 1, 2021 / Accepted: November 18, 2021 / Published online: December 3, 2021

(C) The Author(s) 2021

\section{ABSTRACT}

Introduction: The ObserVational survey of the Epidemiology, tReatment, and Care Of MigrainE study in Japan (OVERCOME [Japan]) assessed the impact and burden of migraine in Japan.

Methods: OVERCOME (Japan) was a cross-sectional, observational, population-based web

Supplementary Information The online version contains supplementary material available at https:// doi.org/10.1007/s40120-021-00305-9.

Y. Matsumori

Sendai Headache and Neurology Clinic, Sendai, Japan

K. Ueda $(\bowtie) \cdot$ M. Komori

Japan Drug Development and Medical Affairs, Eli Lilly Japan K.K., 5-1-28, Isogamidori, Chuo-ku, Kobe-shi 651-0086, Japan

e-mail: ueda_kaname@lilly.com

\section{A. J. Zagar · Y. Kim}

Real World and Access Analytics, Eli Lilly and

Company, Indianapolis, USA

D. H. Jaffe

Real World Evidence, Cerner Enviza (Formerly

Kantar Health), Tel Aviv, Israel

T. Takeshima

Department of Neurology Headache Center,

Tominaga Hospital, Osaka, Japan

K. Hirata

Department of Neurology, Dokkyo Medical

University, Mibu, Japan survey of Japanese people with migraine conducted between July and September 2020. The burden and impact of migraine were assessed using the Migraine Disability Assessment (MIDAS), Migraine-Specific Quality-of-Life Questionnaire (MSQ), Migraine Interictal Burden Scale (MIBS-4), and Work Productivity and Activity Impairment-Migraine scale. Results were stratified by average number of monthly headache days $(0-3,4-7,8-14, \geq 15)$.

Results: In total, 17,071 Japanese people with migraine completed the survey. Of these, 14,033 (82.2\%) met International Classification of Headache Disorders, 3rd edition criteria for migraine and 9667 (56.6\%) reported a physician diagnosis of migraine. Overall, $20.7 \%$ of respondents experienced moderate-to-severe disability (MIDAS). Moderate-to-severe interictal burden (MIBS-4) was experienced by $41.5 \%$ of respondents. MSQ scores in all domains were lowest in respondents with the most frequent headaches ( $\geq 15$ monthly headache days) and highest in those with the lowest frequency headaches ( $\leq 3$ monthly headache days), indicating poorer quality of life in those with more frequent headaches. Work time missed due to migraine (absenteeism) increased with increasing headache frequency, from 3.8 to $6.2 \%$; presenteeism affected $29.8-49.9 \%$ of work time. Although migraine burden was greatest in people with the most frequent headaches, those with the lowest headache frequency still experienced substantial disability, interictal burden, 
and impacts on productivity and quality of life. There was also substantial unmet need for migraine care: $36.5 \%$ of respondents had ever hesitated to seek medical care for their headaches, and $89.8 \%$ had never used preventive medication.

Conclusion: In Japan, the burden of migraine and barriers to migraine care are substantial. Improving patient awareness and healthcare provider vigilance may help improve patient outcomes.

Keywords: Drug therapy; Headache disorders; Japan; Migraine burden; Migraine disorders; Quality of life; Work productivity

\section{Key Summary Points}

\section{Why carry out this study?}

There is a lack of up-to-date knowledge of the impact of migraine in Japanese people with migraine.

The ObserVational survey of the Epidemiology, tReatment, and Care Of MigrainE study in Japan (OVERCOME [Japan]), a cross-sectional observational study, assessed the current status of migraine symptomatology, burden, and care patterns in a representative sample of Japanese people.

\section{What was learned from the study?}

Migraine burden was greatest in people with the most frequent headaches, although substantial disability, interictal burden, and activity impairment were reported even among those with low headache frequency.

Of OVERCOME (Japan) respondents, $36.5 \%$ hesitated to seek medical care for their headaches, and $89.8 \%$ never used a migraine preventive medication.

Improving patient and healthcare provider awareness may help improve patient outcomes.

\section{INTRODUCTION}

Migraine, a chronic neurological disease, is the second most common cause of years lived with disability worldwide [1]. Migraine is known to have substantial impacts on quality of life, everyday function, familial relationships, and work productivity [2-4]. In Japan, where migraine is estimated to affect $6.0-8.6 \%$ of the population [5-7], the individual, economic, and societal impacts of migraine are starting to be recognized $[8,9]$. The economic impact of headache (including migraine), combined with the relative inaccessibility of specialist medical care for many patients [4], has led to a proposed structured framework for headache health care that appears to be both effective and cost-effective for migraine (using European countries as a model $[10,11])$.

Two population-based studies of migraine have been conducted in Japan, both conducted over 20 years ago [5,6]. These surveys revealed a high burden of disease among Japanese people with migraine, including substantial proportions of people requiring bed rest during migraine attacks, experiencing moderate-tosevere impacts on social activity, requiring time off work, and experiencing a general perception of poor health [5, 6]. More recently, an analysis of data from the Japan National Health and Wellness Survey (NHWS) in 2017 revealed that people with a migraine diagnosis had poorer health-related quality of life (HRQoL) and more impairment to work productivity than matched controls without migraine $[12,13]$. A recent workplace-based survey conducted in a Japanese information technology company revealed a high prevalence and burden of migraine among employees and substantial associated economic costs [9]. Although these four studies provide some evidence of the migraine burden in Japan, there is a lack of up-to-date knowledge of the impact of migraine in the Japanese population.

The ObserVational survey of the Epidemiology, tReatment, and Care Of MigrainE study in Japan (OVERCOME [Japan]), a cross-sectional observational study, assessed symptomatology, burden, and care patterns in a representative 
sample of the Japanese population. The study design and broad-scale epidemiological data from the OVERCOME (Japan) study have been published previously [7]. Here we describe in detail the burden of migraine and barriers to migraine care in Japan revealed by OVERCOME (Japan). Migraine burden was assessed using validated measures including the Migraine Disability Assessment (MIDAS), Migraine-Specific Quality-of-Life Questionnaire (MSQ), Migraine Interictal Burden Scale (MIBS-4), and Work Productivity and Activity Impairment-Migraine (WPAI-M) scale.

\section{METHODS}

\section{Study Design and Study Participants}

The OVERCOME (Japan) study was a cross-sectional online survey of adults with and without migraine in Japan, conducted between July and September 2020. This article reports on the OVERCOME (Japan) data for people with migraine only. Ethical approval for the study was granted by the Research Institute of Healthcare Data Science Ethical Review Board (ID: RI2020003). All survey respondents provided informed consent, and all data were anonymized before analysis.

OVERCOME (Japan) was similar in study design to the earlier OVERCOME (US) observational study [14], and the design has been previously described [7]. Briefly, the survey consisted of three stages. In Stage I, a respondent sample representative of the Japanese population was selected using preset demographic sample size quotas for sex, age, and geographic region (quotas based on Japanese census data [7]). In Stage II, people with migraine and severe headache were identified according to the following criteria: at least one headache in the previous 12 months not associated with another illness, head injury, or a hangover; and either met modified criteria for migraine from the International Classification of Headache Disorders, 3rd edition (ICHD-3) [15] or had a self-reported physician diagnosis of migraine. The modified ICHD-3 criteria were the same as those used in the American
Migraine Study [16] and the American Migraine Prevalence and Prevention Study [17]. Stage III of the study assessed migraine symptoms, impact and burden, and healthcare patterns in the migraine group.

As previously described [7], participants were included in the survey if they were adults resident in Japan, members of the Kantar Profiles (Lightspeed) online global survey panel or its panel partners, with access to the internet, able to read and write Japanese, and able to provide electronic informed consent.

\section{Measurements}

\section{Demographics and Clinical Features of Migraine}

Demographic data reported in this manuscript (collected in Stage I) include age, sex, marital status, and employment status. Clinical features of migraine from the Stage III Migraine Assessment Survey reported in the current article include migraine diagnosis type (met ICHD-3 criteria, self-reported diagnosis by a physician, or both) and age at migraine diagnosis.

\section{Migraine Burden}

The impact of headache-related disability was assessed using the MIDAS scale. The MIDAS consists of five items that reflect days an individual missed or experienced reduced productivity at work, home, or social events over a 3 -month period $[18,19]$. Higher MIDAS scores indicate more disability. Four categorical grades are often reported $[18,19]$, based on the total MIDAS score: no or little disability (MIDAS score 0-5), mild disability (MIDAS score 6-10), moderate disability (MIDAS score 11-20), and severe disability (MIDAS score $\geq 21$ ). MIDAS scores are correlated with physicians' assessments regarding the need for medical care, and the MIDAS has demonstrated reliability and validity $[18,19]$. The MIDAS also includes a patient-reported measure of average headache pain severity using an 11-point numerical rating scale, from 0 (no pain at all) to 10 (pain as bad as it could be). The Japanese language version of the MIDAS has been validated [20]. 
Migraine-related HRQoL was measured using the MSQ, version 2.1. The MSQ v.2.1 consists of 14 items using a 6-point Likert-type scale (none of the time, a little bit of the time, some of the time, a good bit of the time, most of the time, and all of the time); the recall period for the questionnaire is the previous 4 weeks [21]. Raw scores were calculated for each of three domains. Role Function-Restrictive (MSQ-RFR) measures the functional impact of migraine through limitations on daily social and work activities; Role Function-Preventive (MSQ-RFP) measures impact through prevention of daily work and social activities; Emotional Function (MSQ-EF) assesses the emotional impact of migraine. Raw scores are converted to a 0-100 scale, with higher scores indicating a better HRQoL [21, 22]. The MSQ is considered reliable, valid, and sensitive to change in migraine [22], and clinically meaningful differences for each domain are well established and widely used [23]. The questionnaire has been validated in Japanese [24, 25].

Interictal burden was measured using the MIBS-4. The MIBS- 4 is a validated four-item instrument that measures the burden related to migraine in the time between migraine attacks (interictal period) [26, 27]. The four items address disruption at work and school, diminished family and social life, difficulty planning, and emotional difficulty. The MIBS-4 specifically asks about the effect of migraine over the previous 4 weeks on days without a headache attack. Response options for each item (don't know/not applicable, never, rarely, some of the time, much of the time, or most or all of the time) have an associated numerical score. Responses are summed across all four items to give a total score ranging from 0 to 12 . The level of interictal burden is categorized into four groups: none (score of 0 ), mild (score of 1-2), moderate (score of 3-4), and severe (score $\geq 5$ ) $[26,27]$.

Work productivity impacts were assessed using the WPAI-M questionnaire [28]. The WPAI itself measures the impact of a specific health problem (in this case, migraine) on work productivity and regular activities over the previous 7 days [28]. The validated questionnaire contains six items (employment status, hours missed from work due to the health problem, hours missed from work for other reasons, hours actually worked, the degree to which health affected work productivity, and the degree to which health affected productivity in regular unpaid activities). The responses are used to calculate four WPAI-M scores: absenteeism, presenteeism, total work productivity impairment, and total activity impairment, which are calculated as impairment percentages, with higher numbers indicating worse outcomes (greater impairment and less productivity) [28].

\section{Medical Care for Migraine}

Healthcare professional consultation and medication use have been previously reported in detail [7]. Here, we report the proportion of respondents who ever sought medical care for migraine or severe headache and those who sought care in the previous year. Respondents were also asked if they had ever hesitated to seek medical care from their doctor or healthcare provider for migraine or severe headache. Those who answered "yes" were asked to give the reason(s) for their hesitation. Respondents could select multiple reasons for hesitation (Table S1 in the electronic supplementary material).

We also report current medication use for acute (both prescription and over-the-counter) and preventive treatment (prescription only) for migraine. Current use was defined as "currently using or typically keep on hand" for acute medications and "taken or used in the last three months" for preventives. Medications for acute treatment of migraine listed in the survey included specific names (brand/generic) of triptans, ergotamine, nonsteroidal anti-inflammatory drugs, and simple/combination analgesics. Preventive medications for migraine listed in the survey included specific names (brand/generic) of antidepressants, antiseizure medications, and antihypertensive medications. Current users of preventive medications were asked to estimate the number of days in the past 30 days that they had been able to take and remembered to take their preventive medication. Respondents were also asked if they had ever used preventive treatment for migraine or 
severe headache; those who had never used preventive medication were asked their reason(s) for not using preventives. Respondents could select multiple reasons (Table S2 in the electronic supplementary material). Respondents who indicated that they had used preventive medication in the past were asked how many preventive medications they had used, when they last took their most recent preventive medication, how long they had used their most recent preventive medication, and why they had stopped taking their most recent preventive medication (multiple reasons could be selected; Table S3 in the electronic supplementary material).

\section{Statistical Analyses}

Mean and standard deviation or median and range are reported for continuous variables, and frequencies and percentages are reported for categorical variables. Results are stratified by monthly headache days (averaged across the preceding 3 months; 0.00-3.99 [0-3], 4.00-7.99 [4-7], 8.00-14.99 [8-14], and $\geq 15.00$ [ $\geq 15]$ ). The four monthly headache days categories were selected to better describe differences and similarities between people with low-frequency episodic migraine (LFEM; 0-3), moderate-frequency episodic migraine (MFEM; 4-7), highfrequency episodic migraine (HFEM; 8-14), and chronic migraine (CM; $\geq 15$ days) [14]. For continuous variables, analysis of variance was used to assess any difference among monthly headache days groups for the respondents' migraine-related assessments and patient-reported outcomes. Chi-square tests were employed to test the association between categorical variables and monthly headache days. No multiplicity adjustment was made in the statistical testing, and $p<0.05$ was considered significant. Statistical analyses were performed using SAS Enterprise Guide 7.15 (SAS Institute, Cary, NC, USA).

\section{RESULTS}

\section{Demographics and Clinical Features of Migraine}

The total number of respondents who completed the migraine survey was 17,071 . As previously reported, the mean age of respondents was 40.7 years, and $66.5 \%$ were female [7]. On average, respondents with more frequent headaches (HFEM and CM groups) were older than those with fewer headaches (Table 1). The proportion of female respondents differed across the monthly headache days groups, with the highest proportion (73.3\%) seen in the HFEM group. The percentage of respondents who were married and the percentage who were employed decreased as monthly headache frequency increased.

The proportion of respondents who met ICHD-3 criteria for migraine increased with increasing headache frequency, from $80.8 \%$ in the LFEM group to $87.1 \%$ in the CM group. A similar pattern was seen in self-reported diagnoses of migraine by a physician, which increased with increasing headache frequency, from $52.7 \%$ in the LFEM group to $68.5 \%$ in the $\mathrm{CM}$ group. However, for these diagnosed respondents, the mean age of migraine diagnosis was not different among the monthly headache days groups. The overall mean age at diagnosis was 26.4 years.

Average pain severity was 5.1 (scale maximum 10), and pain severity increased with increasing headache frequency (Table 1).

\section{Migraine Burden}

Severity of disability (MIDAS score) varied with headache frequency (Fig. 1a; $p<0.001$ ). A higher proportion of people in the MFEM, HFEM, and CM groups (31.9-51.0\%) experienced moderate-to-severe disability than in the LFEM group (11.5\%). MSQ scores in all three domains differed across the monthly headache days groups (Fig. $1 \mathrm{~b} ; p<0.001$ ). MSQ-RFR, MSQ-RFP, and MSQ-EF were lowest in the CM ( $\geq 15$ headache days per month) group, 
Table 1 Demographic characteristics and clinical features of survey respondents

\begin{tabular}{|c|c|c|c|c|c|c|}
\hline \multirow{2}{*}{$\begin{array}{l}\text { Demographic } \\
\text { characteristics }\end{array}$} & \multirow{2}{*}{$\begin{array}{l}\text { Total }^{\mathrm{a}} \\
(N=17,071)\end{array}$} & \multicolumn{4}{|c|}{ Monthly headache days category } & \multirow[t]{2}{*}{$p$ value $^{\mathrm{b}}$} \\
\hline & & $\begin{array}{l}\text { LFEM, 0-3 } \\
(n=11,498)\end{array}$ & $\begin{array}{l}\text { MFEM, } 4-7 \\
(n=2714)\end{array}$ & $\begin{array}{l}\text { HFEM, 8-14 } \\
(n=1608)\end{array}$ & $\begin{array}{l}\mathrm{CM}, \geq 15 \\
(n=1251)\end{array}$ & \\
\hline Age, years, mean (SD) & $40.7(13.0)$ & $40.3(13.0)$ & $40.8(12.8)$ & $42.2(13.1)$ & $43.0(12.7)$ & $<0.001^{\mathrm{c}}$ \\
\hline Female, $n(\%)$ & $11,354(66.5)$ & $7,325(63.7)$ & $1,958(72.1)$ & $1,178(73.3)$ & $893(71.4)$ & $<0.001^{\mathrm{d}}$ \\
\hline Married, $n(\%)$ & $8,555(50.1)$ & $5846(50.8)$ & $1343(49.5)$ & $797(49.6)$ & $569(45.5)$ & $<0.001^{\mathrm{d}}$ \\
\hline Employed, $n(\%)$ & $12,383(72.5)$ & $8,462(73.6)$ & $1,965(72.4)$ & $1,131(70.3)$ & $825(65.9)$ & $<0.001^{\mathrm{d}}$ \\
\hline \multicolumn{7}{|l|}{ Migraine diagnosis, $n(\%)$} \\
\hline Met ICHD-3 criteria & $14,033(82.2)$ & $9,292(80.8)$ & $2,281(84.1)$ & $1,371(85.3)$ & $1,089(87.1)$ & $<0.001^{\mathrm{d}}$ \\
\hline ICHD-3 with diagnosis & $6,629\left(38.8^{\mathrm{e}}\right)$ & $3,851\left(33.5^{\mathrm{e}}\right)$ & $1,243\left(45.8^{\mathrm{e}}\right)$ & $840\left(52.2^{\mathrm{e}}\right)$ & $695\left(55.6^{\mathrm{e}}\right)$ & $<0.001^{\mathrm{d}}$ \\
\hline $\begin{array}{l}\text { Self-reported diagnosis by a } \\
\text { physician }\end{array}$ & $9,667(56.6)$ & $6,057(52.7)$ & $1,676(61.8)$ & $1,077(67.0)$ & $857(68.5)$ & $<0.001^{\mathrm{d}}$ \\
\hline $\begin{array}{l}\text { Age at migraine diagnosis, } \\
\text { years, mean }(\mathrm{SD})\end{array}$ & $26.4(11.7)$ & $26.2(11.3)$ & $26.7(11.9)$ & $26.8(12.2)$ & $26.9(12.4)$ & $0.341^{\mathrm{c}}$ \\
\hline Pain severity, mean (SD) & $5.1(2.2)$ & $4.8(2.2)$ & $5.5(1.9)$ & $5.9(1.8)$ & $6.4(1.8)$ & $<0.001^{\mathrm{c}}$ \\
\hline \multicolumn{7}{|l|}{ Consultation patterns ${ }^{\mathrm{f}}, n(\%)$} \\
\hline Ever sought care for migraine & $9,800(57.4)$ & $6,025(52.4)$ & $1,719(63.3)$ & $1,122(69.8)$ & $934(74.7)$ & $<0.001^{\mathrm{d}}$ \\
\hline $\begin{array}{l}\text { Sought care for migraine in } \\
\text { the previous year }\end{array}$ & $6,774(39.7)$ & $3,930(34.2)$ & $1,226(45.2)$ & $866(53.9)$ & $752(60.1)$ & $<0.001^{\mathrm{d}}$ \\
\hline \multicolumn{7}{|l|}{ Current medication use, $n(\%)$} \\
\hline $\begin{array}{l}\text { Acute treatment } \\
\text { prescription/OTC } \\
\text { medication }^{\mathrm{g}}\end{array}$ & $14,869(87.1)$ & $9,802(85.3)$ & $2,465(90.8)$ & $1,466(91.2)$ & $1,136(90.8)$ & $<0.001^{\mathrm{d}}$ \\
\hline Preventive treatment ${ }^{\mathrm{h}}$ & $1,567(9.2)$ & $854(7.4)$ & $274(10.1)$ & $234(14.6)$ & $205(16.4)$ & $<0.001^{\mathrm{d}}$ \\
\hline
\end{tabular}

$A N O V A$ analysis of variance, $C M$ chronic migraine, HFEM high-frequency episodic migraine, ICHD-3 International Classification of Headache Disorders, 3rd edition, LFEM low-frequency episodic migraine, $M F E M$ moderate-frequency episodic migraine, $O T C$ over-the-counter, $S D$ standard deviation

${ }^{\text {a }}$ Previously reported [7]

${ }^{\mathrm{b}}$ Comparisons across all the migraine headache days groups

${ }^{c} p$ value from ANOVA ( $F$-test)

$\mathrm{d}_{p}$ value from chi-square test

${ }^{\mathrm{e}}$ Percentages shown are proportions of total migraine and monthly headache days groups. Proportions of those who met the ICHD-3 criteria who also had a diagnosis of migraine were: Total 47.2\%; LFEM 41.4\%; MFEM 54.5\%; HFEM 61.3\%; CM $63.8 \%$

${ }^{\mathrm{f}}$ Sites of care for migraine included primary care/internist clinics, neurology clinics (including headache specialists), and neurosurgeon clinics [7]

gIncluded triptans, ergotamine, nonsteroidal anti-inflammatory drugs, and simple/combination analgesics

${ }^{\mathrm{h}}$ Included antidepressants, antiseizure medications, and antihypertensive medications 
(a)

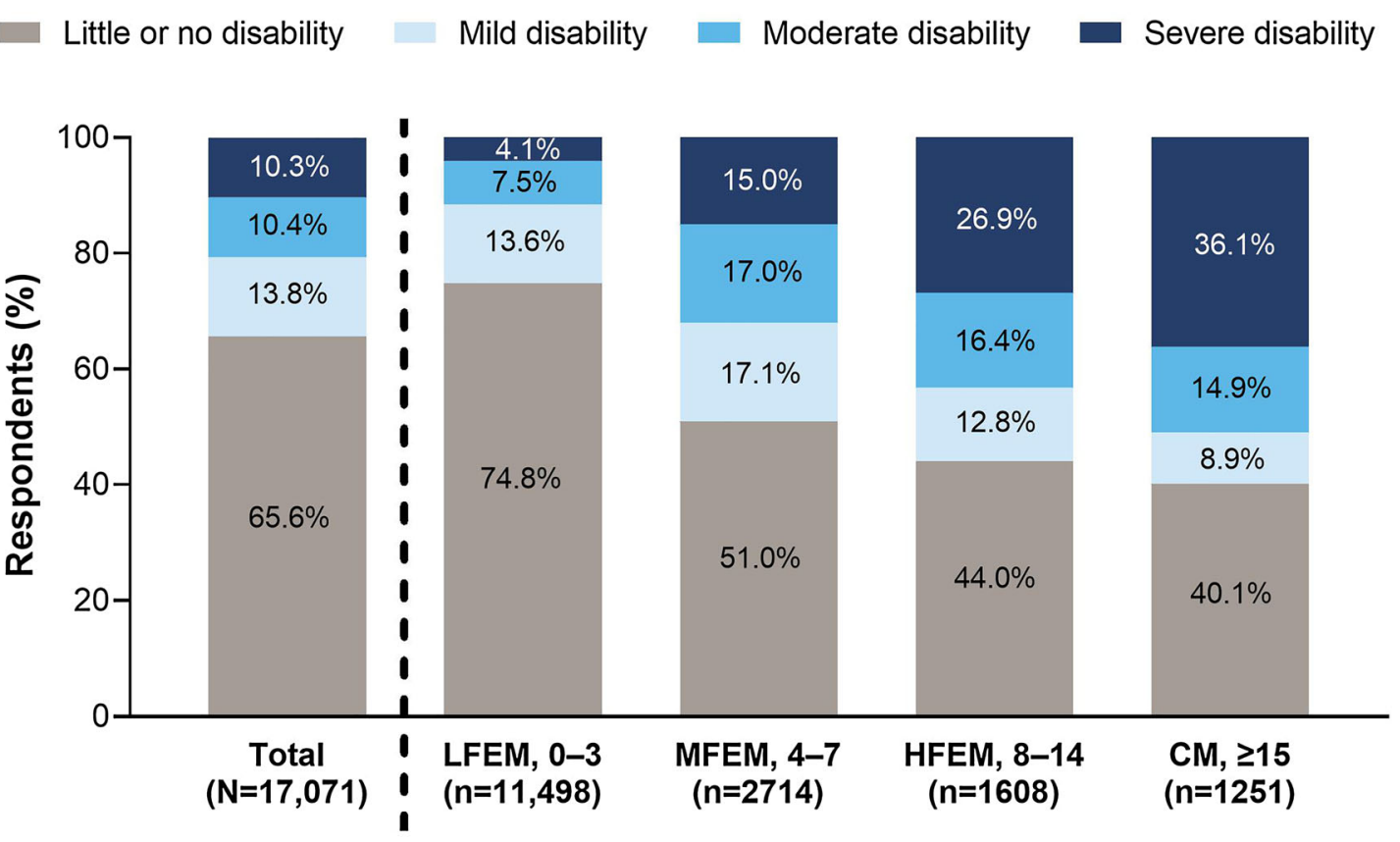

\section{Monthly headache days group}

(b)

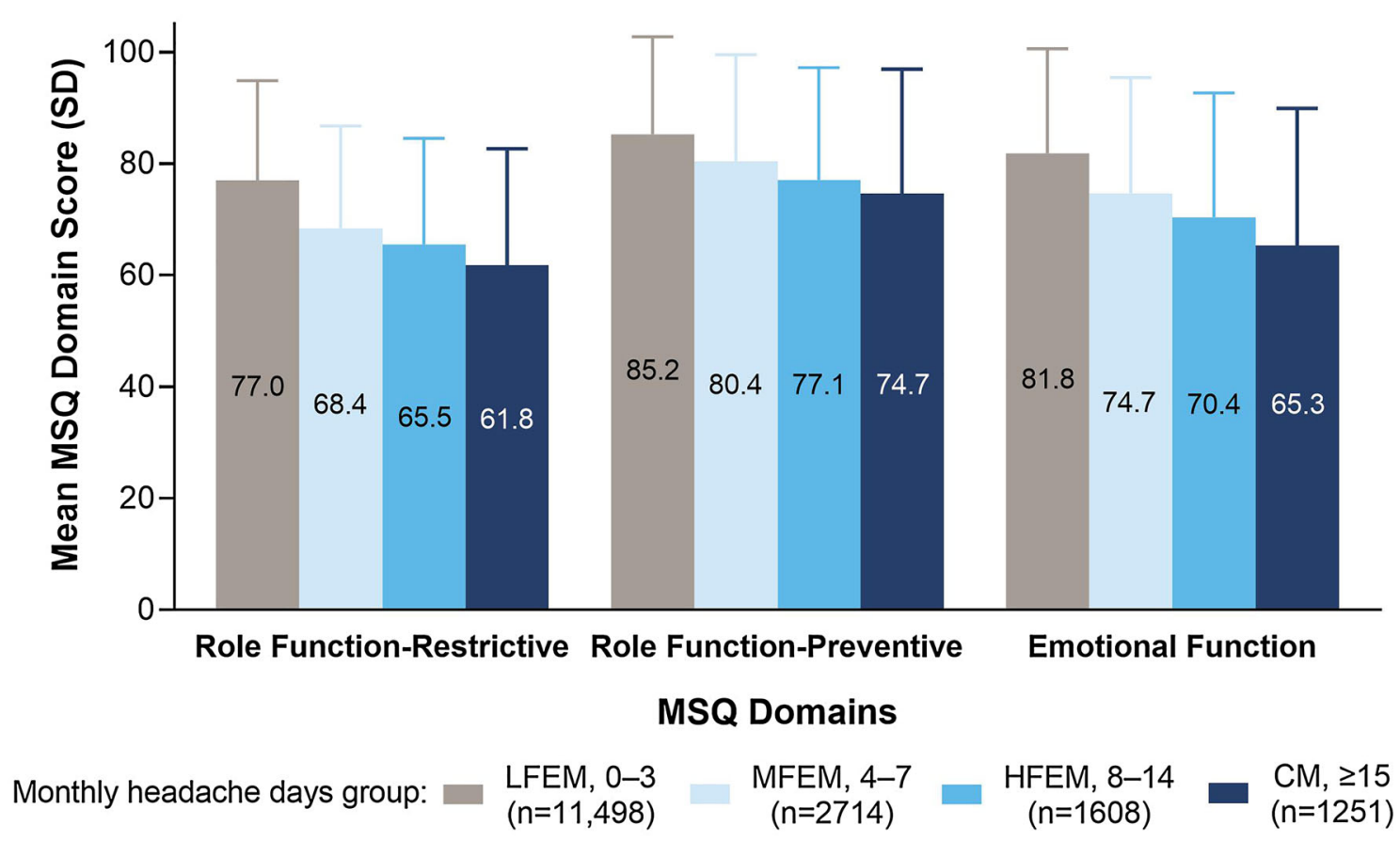

Fig. 1 a Migraine-related disability (MIDAS grade) in each of the monthly headache days groups. b HRQoL scores (MSQ domain scores) in each of the monthly headache days groups. CM, chronic migraine; HFEM, high-frequency episodic migraine; HRQoL, health-related quality of life; LFEM, low-frequency episodic migraine;
MFEM, moderate-frequency episodic migraine; MIDAS, Migraine Disability Assessment; MSQ, Migraine-Specific Quality-of-Life Questionnaire 


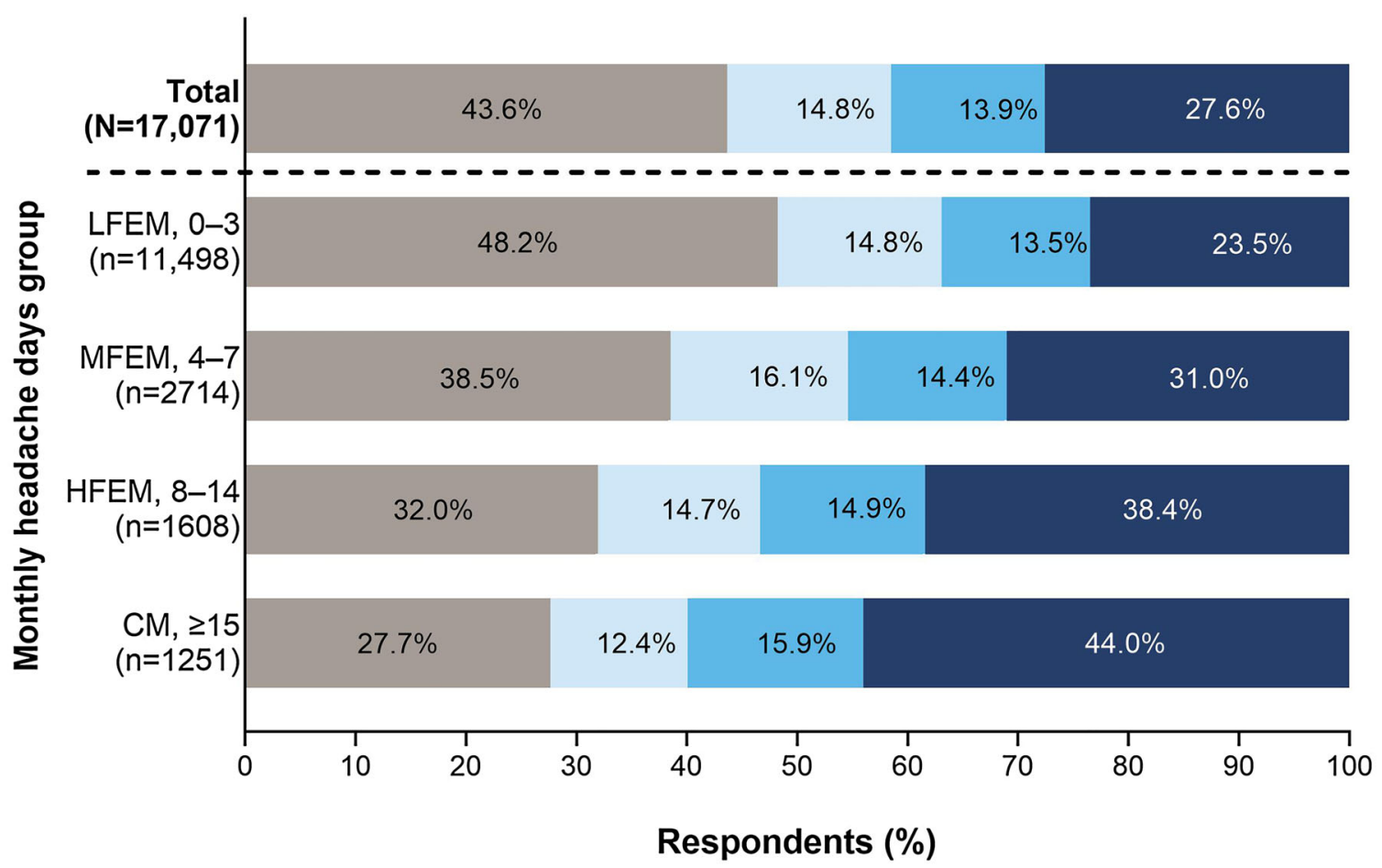

Fig. 2 Interictal burden across monthly headache days groups. Interictal burden categories from the MIBS-4 are none (score of 0 ), mild (score of 1-2), moderate (score of 3-4), and severe (score $\geq 5$ ). CM, chronic migraine; HFEM, high-frequency episodic migraine; LFEM, low-

indicating poorer HRQoL in those with the highest frequency of headaches.

Overall, the interictal burden of migraine was substantial, with $41.5 \%$ of patients experiencing moderate-to-severe burden between migraine attacks (Fig. 2). Even in the LFEM group, about half $(51.8 \%)$ of respondents experienced at least some interictal burden. The interictal burden categories varied significantly with headache frequency $(p<0.001)$. Severe interictal burden was experienced by a greater proportion of people as headache frequency increased. In the HFEM and CM groups, 53-60\% of respondents experienced moderate-to-severe interictal burden.

As previously reported, overall absenteeism (mean percentage of work time missed) and presenteeism (percentage of work time impaired by migraine) were $4.2 \%$ and $34.3 \%$, respectively [7]. The impact of migraine on work frequency episodic migraine; MFEM, moderate-frequency episodic migraine; MIBS-4, Migraine Interictal Burden Scale

productivity increased with increasing headache frequency (Fig. 3; $p<0.001$ for all workrelated subscales), with presenteeism as high as $50 \%$ in the CM group. Total activity impairment for regular unpaid activities also increased significantly with increasing headache frequency (Fig. 3; $p<0.001$ ); in the HFEM and CM groups, total activity was impaired $48-53 \%$ of the time compared with $31-42 \%$ in the LFEM and MFEM groups.

\section{Medical Care for Migraine}

Overall, $57.4 \%$ of respondents had ever sought care for their migraine or severe headache; $39.7 \%$ had sought care in the previous year (Table 1). The proportion of respondents who had sought care increased as headache frequency increased. 


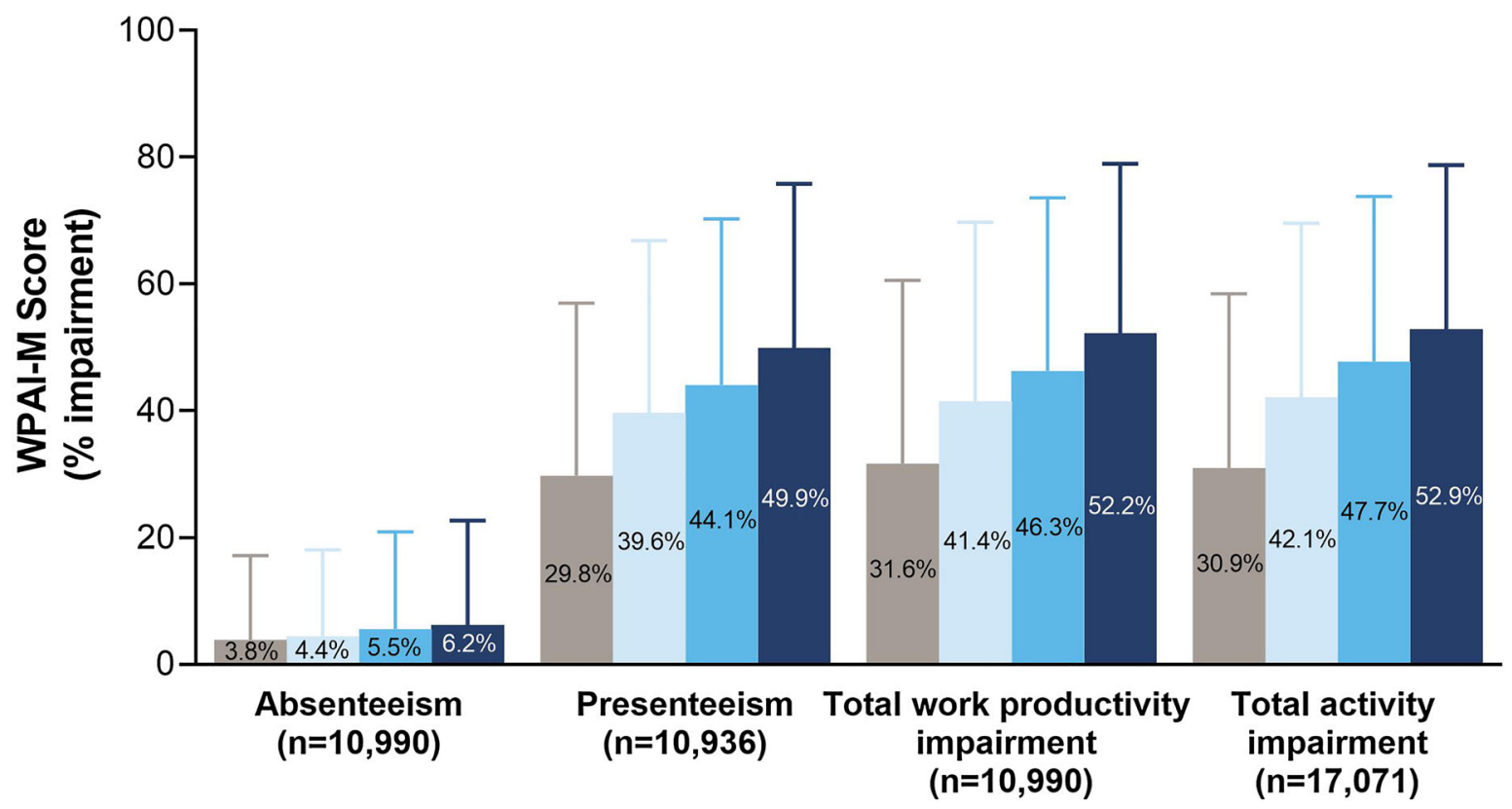

WPAI-M Sub-scales

\section{Monthly headache days group: LFEM, 0-3}

Fig. 3 WPAI-M scores across monthly headache days groups. All respondents answered the WPAI-M total activity impairment questions, but only those who were full-time, part-time, or self-employed provided data for absenteeism, presenteeism, and overall work impairment. The numbers of respondents in each monthly headache days category for each of the WPAI-M subscales were: absenteeism LFEM $n=7295$, MFEM $n=1854$, HFEM $n=1049$, CM $n=792$; presenteeism LFEM $n=7261$, $\operatorname{MFEM} n=1848, \operatorname{HFEM} n=1043, \mathrm{CM} n=784$; total

Overall, $36.5 \%$ of respondents had ever hesitated to seek care for their migraine or severe headache (Fig. 4a). The proportion of respondents who hesitated to seek care increased significantly $(p<0.001)$ with increasing headache frequency. The most common reasons given for hesitation in seeking care for migraine or severe headache were: "I felt my migraine or headache would not be taken seriously," "I did not think they were serious/painful enough," and "I did not know where to find a doctor or healthcare provider who treated them." The proportion of respondents who gave each of these reasons varied significantly across the monthly headache days groups (Fig. 4b, $p<0.001$ for each reason).
MFEM, 4-7 HFEM, 8-14

$\mathrm{CM}, \geq 15$

work impairment LFEM $n=7295$, MFEM $n=1854$, HFEM $n=1049$, CM $n=792$; total activity impairment LFEM $n=11,498$, MFEM $n=2714$, HFEM $n=1608$, CM $n=1251$. CM, chronic migraine; HFEM, highfrequency episodic migraine; LFEM, low-frequency episodic migraine; MFEM, moderate-frequency episodic migraine; WPAI-M, Work Productivity and Activity Impairment-Migraine

Additional reasons given by respondents for hesitation in seeking care are provided in Table S1 in the electronic supplementary material.

Current use of acute medications was similar in the MFEM, HFEM, and CM groups ( 91\% of respondents) and lower in the LFEM group $(85.3 \%)$ (Table 1). Current use of preventive medications was more common in the HFEM and CM groups (14.6-16.4\% of respondents) compared with the LFEM and MFEM groups (Table 1). Among current users of preventive medications, the average number of days that medication was taken in the previous 30 days was 10.9 days (7.4 days in the LFEM group; 
(a)

- Hesitated in Seeking Care

No Hesitation in Seeking Care

Don't Remember

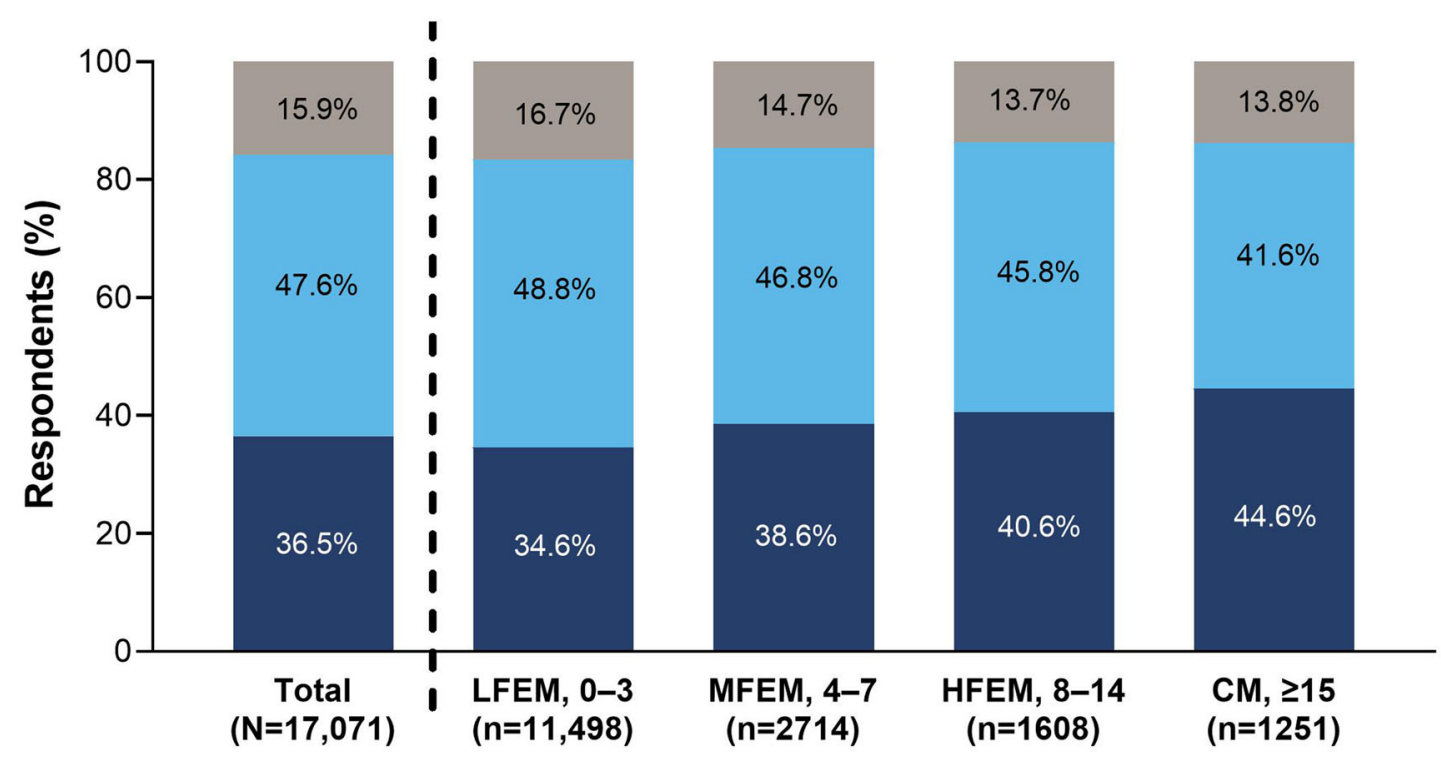

Monthly headache days group

(b)

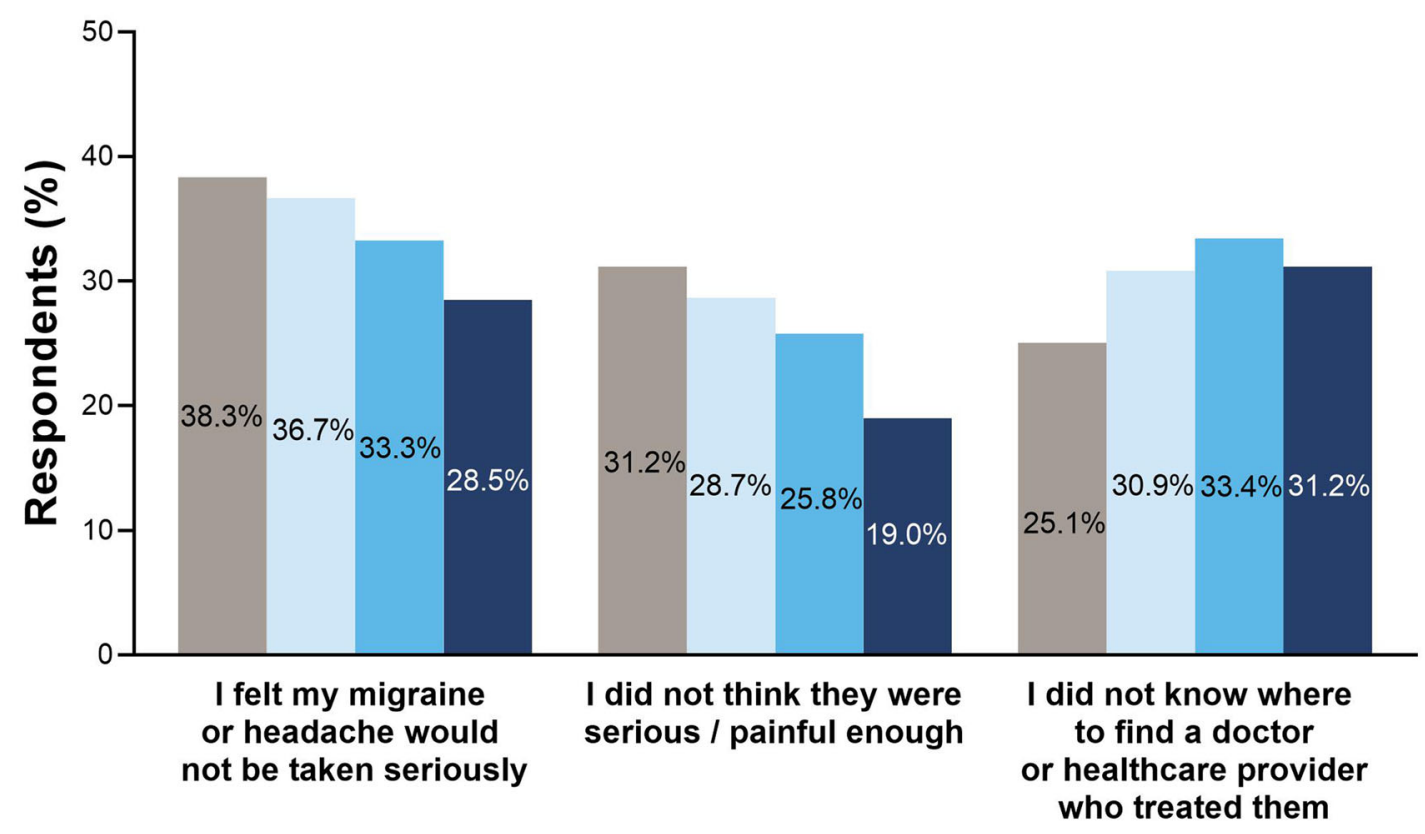

Monthly headache days group:

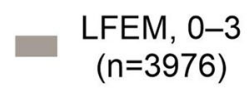
MFEM, 4-7 $(n=1047)$
HFEM, 8-14 $(n=652)$

$\mathrm{CM}, \geq 15$

$(n=558)$ 
4Fig. 4 Hesitation in seeking care for migraine across monthly headache days groups: a proportion of respondents who had and had not hesitated to seek care for migraine from their doctor or healthcare provider and b frequency of the top three reasons for not seeking care for migraine or severe headache. CM, chronic migraine; HFEM, high-frequency episodic migraine; LFEM, lowfrequency episodic migraine; MFEM, moderate-frequency episodic migraine

18.5 days in the CM group), indicating low treatment adherence.

Most respondents had never tried preventive medications (Fig. 5a), including in the $\mathrm{CM}$ group ( $\geq 15$ headache days per month), in which $81.1 \%$ of respondents had never used preventive medication. In the LFEM group, $4.7 \%$ had tried two or more preventive medications; this increased to $9.6 \%$ in the CM group. Among those who had never tried preventive medication, common reasons for not having used preventive medication included: "The other medications I use work well enough," "My migraines/severe headaches are not that bad/ serious," "I am concerned about the side effects of medication/the effects of using them too often," "It costs too much/I do not want to spend the money," and "I did not know there were prescription medications that would prevent/reduce migraine/severe headaches." The proportion of respondents who gave each of these reasons varied significantly across the monthly headache days groups (Fig. 5b, $p<0.001$ for each reason). Additional reasons given by respondents for not using preventive migraine treatment are provided in Table S2 in the electronic supplementary material. Among respondents who had tried and stopped preventive medication, $53.1 \%$ had stopped taking the most recent medication $>2$ years ago, and $54.3 \%$ had taken the medication for $\leq 6$ months before stopping (Table S3 in the electronic supplementary material). The most common reason for discontinuing preventive medication was that it was not working $(24.4 \%$ of respondents).

\section{DISCUSSION}

OVERCOME (Japan) is the first nationwide, population-based, demographically representative survey to describe migraine symptomatology, burden, and care patterns in Japan. Among study participants, the burden of migraine and severe headache was substantial. Although greater impacts were seen in people with the most frequent headaches, even people with the lowest headache frequency experienced substantial disability, interictal burden, and impacts on productivity and HRQoL. Many of those surveyed were hesitant to seek medical care for their headaches, and most had never taken potentially beneficial preventive medication. These results from the OVERCOME (Japan) survey will help those committed to improving migraine care in Japan identify and address areas of unmet need.

The severity of migraine-related disability, as measured with the MIDAS, increased with increasing headache frequency. Half $(51 \%)$ of the OVERCOME (Japan) respondents with CM experienced moderate-to-severe disability, somewhat lower than in the Adelphi Migraine Disease Specific Programme study of Japanese patients with migraine (60\% of CM patients) [29]. The lower proportion in OVERCOME (Japan) is likely due to it being a population-based study, whereas the Adelphi study sampled a clinical subpopulation. In the current study, $75 \%$ of people with LFEM and $40-50 \%$ of people with MFEM, HFEM, and CM reported little to no disability. These proportions are higher than previously reported in non-Japanese populations. For example, in the International Burden of Migraine Study (IBMS) [3], only $29.4 \%$ of participants reported little to no disability. Differences in reported migraine-related disability between Japanese and non-Japanese populations have been previously noted in clinical trials and may be due in part to cultural differences around perception of pain [30]. Although reports of severe disability may be lower in Japanese populations, this does not lessen the impact on those affected. In OVERCOME (Japan), severe disability was reported by $15 \%$, $27 \%$, and $36 \%$ of respondents in the MFEM, 
(a)

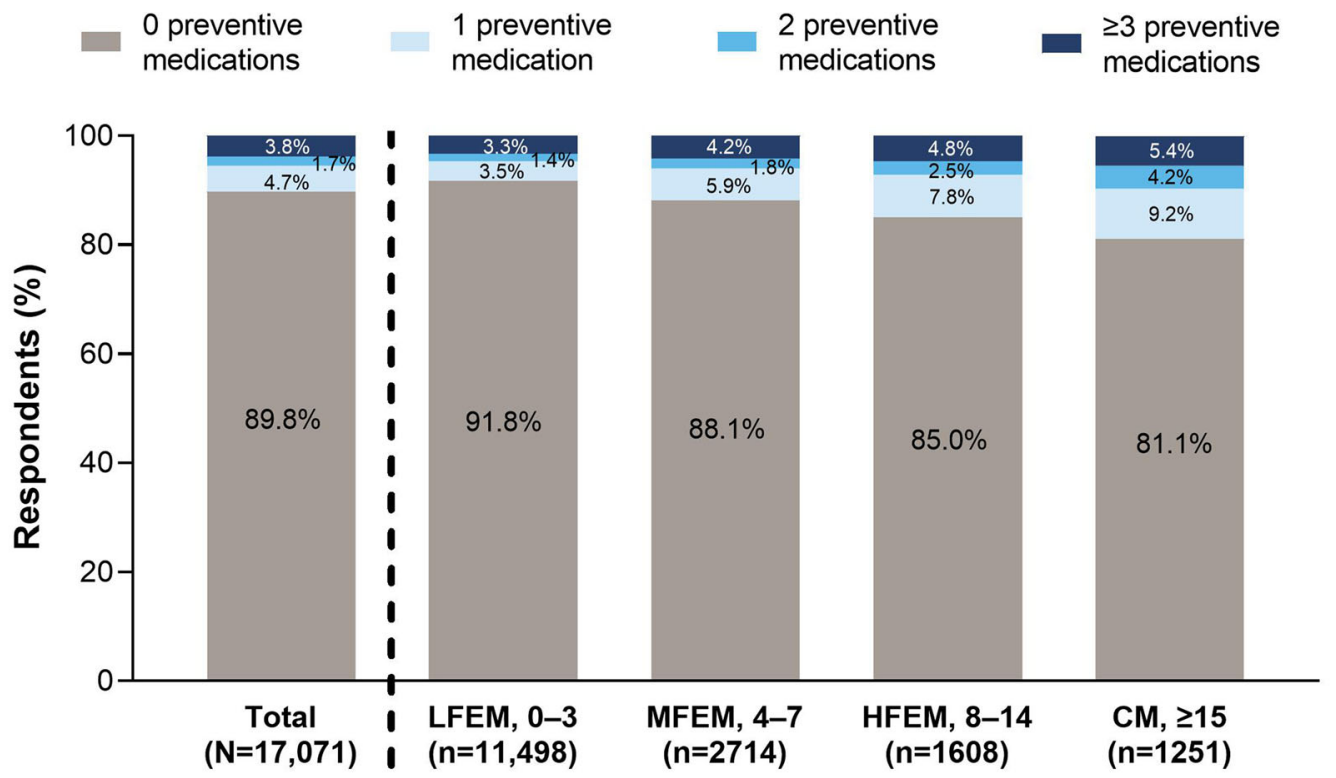

Monthly headache days group

(b)

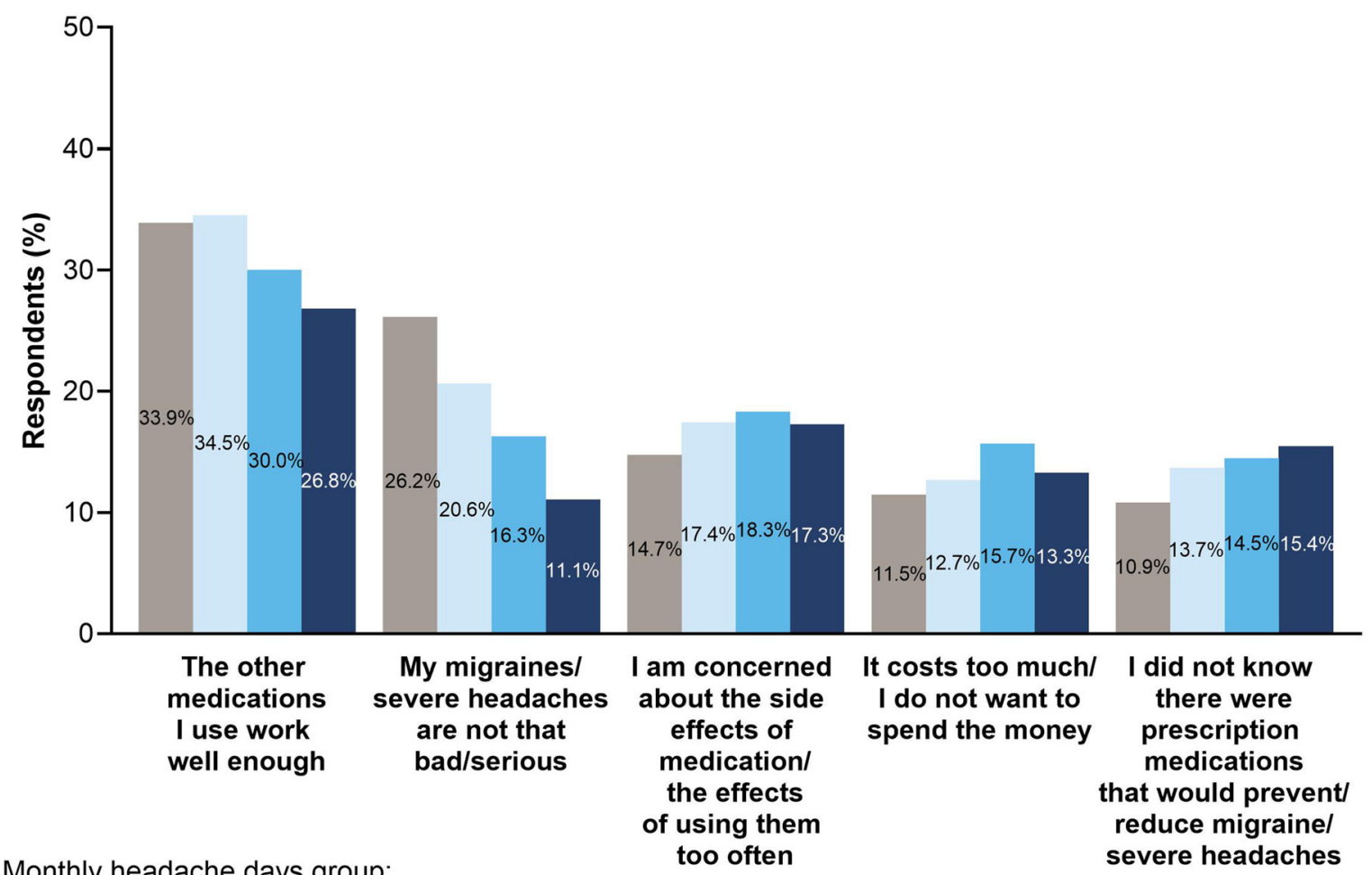

Monthly headache days group:

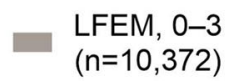
MFEM, 4-7
$(n=2351)$

HFEM, 8-14
$(n=1333)$

$\mathrm{CM}, \geq 15$
$(\mathrm{n}=985)$ 
4Fig. 5 Use of preventive migraine medication across monthly headache days groups: a the number of preventive medications ${ }^{a}$ ever used by respondents and $\mathbf{b}$ frequency of the top five reasons for not having ever used preventive medication. ${ }^{a}$ Respondents who did not answer this question were assumed not to have used preventives, i.e., were included in the "0 preventive medications used" group. CM, chronic migraine; HFEM, high-frequency episodic migraine; LFEM, low-frequency episodic migraine; MFEM, moderate-frequency episodic migraine

HFEM, and CM groups, respectively. Similar patterns were seen in the Adelphi study [29]. These results from OVERCOME (Japan) highlight that people with moderate headache frequencies also experience substantial migrainerelated disability.

The impact on HRQoL, as measured by the MSQ, was also affected by headache frequency. Consistent with the IBMS [3], lower MSQ scores, indicating poorer HRQoL, were associated with higher headache frequencies across all three domains of the MSQ. The MSQ-RFR and MSQEF domains had generally lower scores than the MSQ-RFP domain, indicating that limitations on daily activities and emotional impacts may have a greater effect on HRQoL than on prevention of daily activities. This is consistent with previous studies that used MSQ to assess HRQoL in Japanese people with migraine $[25,31]$ and the IBMS [3].

The OVERCOME (Japan) study provides the first data on psychological, social, and emotional interictal burden in Japanese people with migraine, measured using the MIBS-4. Overall, more than half $(56 \%)$ of the respondents reported that they experienced at least some burden between migraine attacks. This was consistent with a recent employee survey in a Japanese information technology company, in which $40 \%$ of respondents with migraine experienced interictal symptoms, but emotional and social interictal burden was not measured [9]. In OVERCOME (Japan), interictal burden was strongly associated with headache frequency, with $60 \%$ of the CM group experiencing moderate-to-severe interictal burden. However, it is notable that even the LFEM group experienced a substantial interictal burden, with
$>35 \%$ reporting moderate-to-severe burden. It is recommended that people with migraine who report moderate-to-severe interictal burden on the MIBS- 4 are offered additional treatment options, including non-pharmacological strategies for reducing interictal burden and optimization of their pharmacological treatment [27]. Optimization of treatment may include reevaluation of acute medication options and/or addition of preventive medication. The OVERCOME (Japan) study has identified a large proportion of people with migraine who may be candidates for optimization of pharmacological treatments and who could also benefit from non-pharmacological interventions. For example, educational and counseling programs in the workplace, such as the one recently initiated by a Japanese information technology company [32], have been shown to decrease disability and improve disease self-management for people with migraine [33].

Japanese people in the highest headache frequency groups experienced impairment in both workplace and other activities around 50\% of the time, whereas those in the LFEM and MFEM groups were impaired at work and in other activities around $30-40 \%$ of the time. These levels of impairment support the results of the Adelphi study of physicians and their patients with migraine, in which participants with CM and HFEM were impaired at work and in other activities up to $55 \%$ of the time, and those with lower headache frequencies were impaired $30-40 \%$ of the time [29]. Impairment was greater in people with migraine in the OVERCOME (Japan) study than has been reported for people without migraine. In a direct comparison of Japanese people with and without migraine, using data from the Japan NHWS, those without migraine had total work impairment scores of only $20 \%$ and total activity impairment scores of 22\% [12]. WPAI-M absenteeism scores in OVERCOME (Japan) ranged from $3.8 \%$ in the LFEM group to $6.2 \%$ in the CM group. These absenteeism scores were similar to those in Japanese people without migraine $(3.1 \%)$ and with migraine $(7.0 \%)$ in the NHWS analysis [12]. The lower absenteeism rate in the lower headache frequency groups may reflect the influence of the Japanese 
cultural belief in the virtue of hard work (gaman in Japanese; this is typically translated as "perseverance," "patience," "tolerance," "self-control," or "self-denial"). However, despite relatively low absenteeism, the results of OVERCOME (Japan) reveal substantial overall impairment in the workplace and in other regular activities, which has implications for economic assessments of the impact of migraine in Japan. The economic cost of migraine-related presenteeism in Japan, based on data from information technology workers, has been estimated to be up to US\$21.3 billion per year [9].

Overall, $36.5 \%$ of respondents in OVERCOME (Japan) reported that they had hesitated to seek care for migraine or severe headache. Hesitancy to seek medical care increased with increasing headache frequency. This is concerning because those with the most regular headaches are the most in need of intervention. One of the most common reasons for hesitancy, "I did not think they were serious/painful enough" (given by $20-30 \%$ of respondents), suggests that many Japanese people may not fully recognize the impact of migraine on their everyday lives. In an earlier Japanese population study, reasons for not consulting physicians also included "headache not severe enough" (30-36\%) or "headache not serious enough" (7-15\%) [6]. Similarly, the most common reason for not consulting a physician among Japanese information technology workers with headache disorders was the belief that their headache disorder was not serious enough [9]. In OVERCOME (Japan), this reason was most likely to be given by those with lower headache frequencies and least likely by those with the highest frequency, which implies that regularity of headaches is recognized by people with migraine as an indicator of the seriousness of migraine/severe headache. However, it is of concern that nearly one-fifth (19.0\%) of people who experienced migraine or severe headache $\geq 15$ times per month still regarded their headaches as "not serious enough." The most common reason for hesitancy to seek care, "I felt my migraine or headache would not be taken seriously," was reported by around $30-40 \%$ of respondents. This may be due to a lack of confidence or trust in medical practitioners: a recent Wellcome Trust report on global attitudes to science and health found that only $26 \%$ of Japanese people surveyed had a lot of trust in doctors and nurses, substantially lower than in regions such as North America (52\%), Northern Europe (65\%), and Australia/ New Zealand (65\%) [34]. Alternatively, it may reflect an overall dissatisfaction with medical treatment, as has been reported in Japanese people with chronic musculoskeletal pain [35]. A structured headache healthcare system, in which the majority of patients with migraine would be under the care of primary physicians well educated in headache disorders $[4,10,11]$, has the potential to improve trust and reduce dissatisfaction with medical care for migraine.

A large majority (89.8\%) of OVERCOME (Japan) survey respondents had never used preventive medications. In the Adelphi study, which only included people with migraine who were receiving medical care, $30 \%$ of those with $\mathrm{CM}$ and $60 \%$ of those with episodic migraine had never received preventive medications [29]. The higher proportion in OVERCOME (Japan) likely reflects it being a population-based study that included people who were not under current medical care for migraine. The most common reason for not using preventive medication was that people with migraine felt that their current medications worked well enough. However, there were also respondents who believed that their migraine/severe headaches were not sufficiently serious to warrant preventive treatment. Around $10-15 \%$ of respondents did not know about preventive medications. It is particularly concerning that this response was most commonly given by those with the highest headache frequency (15.4\% of respondents in this group). It appears that there may be a need to improve patient education for Japanese people with migraine, particularly to inform them about treatment options for those who experience HFEM or CM. Interestingly, the potential cost of preventive medication was a concern to only a relatively small proportion of respondents $(\sim 12-16 \%)$.

OVERCOME (Japan) also revealed unmet needs among respondents who were currently using or had previously used preventive medication. Treatment adherence to current 
preventive medications was low. In addition, discontinuation rates among those who had previously used preventives were high, with $54.3 \%$ stopping within 6 months and $36.4 \%$ stopping within 3 months. These results are consistent with an analysis of Japanese insurance claims data, in which $62.2 \%$ of migraine patients who initiated preventive treatment discontinued that treatment within approximately 2 months [36]. The most common reason for discontinuation among OVERCOME (Japan) respondents was that the medication was not working, but it is worth noting that over half $(53.1 \%)$ of these previous preventive medication users had last tried a preventive more than 2 years ago. Given that the standard of care for oral preventive medications approved for migraine treatment in Japan is different from other countries [36], education about recent preventive medication options available in Japan would likely be valuable for this group.

The results of OVERCOME (Japan) also highlight the substantial burden of migraine on Japanese women, particularly those in their 20s, $30 \mathrm{~s}$, and 40s. Among the survey-eligible female population aged 18-49 years, the proportion who were in the migraine group ranged from $12.4 \%$ to $15.1 \%$ (data not shown), well above the proportion of migraine cases in the overall eligible population $(7.4 \%$ [6]). In addition, $>70 \%$ of respondents with migraine or severe headache who experienced $\geq 4$ monthly headache days were female. For women in this age group, who are typically busy with their careers and may also have additional family care responsibilities, the disease-specific burden of migraine may have substantial impacts on work productivity and HRQoL.

The OVERCOME (Japan) study is the first population-based epidemiological survey of migraine in Japan in over 20 years. The survey provided the first MIBS-4 data for interictal burden in Japanese people with migraine. The subgroup analysis based on headache frequency is a key strength that allows better insight into the impact of migraine on people with infrequent as well as frequent headaches. Selection bias was reduced by using a sample that was demographically representative of the Japanese population and included respondents with a large variation in migraine burden and headache frequencies. Another strength of the study was the use of patient-reported outcomes, including validated measures wherever possible. Patient-reported outcomes provide data that cannot easily be obtained by alternative realworld evidence methodologies, such as medical records data or insurance claims.

The OVERCOME (Japan) study also has some limitations. There is a possibility of recall bias because all survey data were self-reported, and the low participation rate ( $\sim 7 \%$ of those invited to do the survey [7]) and self-selection by respondents means there was also the potential for participation bias. Despite the use of a quota-sampling method, the OVERCOME (Japan) sample differed slightly from the demography of the Japanese population [7]. These differences (e.g., individuals aged $\geq 65$ years were underrepresented) were probably related to limited accessibility of online surveys to certain demographic groups and may limit the generalizability of the results. However, as migraine is most prevalent in those aged $30-49$ years $[7,37]$, this underrepresentation is unlikely to affect our findings markedly. With respect to the data collected, the migraine diagnoses reported by respondents were not physician verified, and causal inferences between variables cannot be made owing to the cross-sectional survey design.

\section{CONCLUSIONS}

In this large sample of Japanese people with migraine, the burden of migraine and barriers to migraine care were substantial. Improving patient awareness and healthcare provider vigilance may help improve patient outcomes.

\section{ACKNOWLEDGEMENTS}

The authors sincerely thank all study participants. 
Funding. This study was sponsored by Eli Lilly and Company, Indianapolis, IN, USA, and operated by Cerner Enviza (formerly Kantar Health), North Kansas City, MO, USA. Eli Lilly and Company was involved in the study design, data analysis, and preparation of the manuscript. Cerner Enviza (formerly Kantar Health) was involved in the study design, data collection, data analysis, and preparation of the manuscript. The Rapid Service Fee was funded by Eli Lilly Japan K.K.

Medical Writing Assistance. Medical writing assistance was provided by Koa Webster, PhD, and Rebecca Lew, PhD, CMPP, of ProScribe - Envision Pharma Group, and was funded by Eli Lilly Japan K.K. ProScribe's services complied with international guidelines for Good Publication Practice (GPP3).

Authorship. All named authors meet the International Committee of Medical Journal Editors (ICMJE) criteria for authorship for this article, take responsibility for the integrity of the work as a whole, and have given their approval for this version to be published.

Authors' Contributions. All authors participated in the interpretation of study results, and in the drafting, critical revision, and approval of the final version of the manuscript. Yasuhiko Matsumori, Kaname Ueda, Mika Komori, Anthony J. Zagar, Dena H. Jaffe, Takao Takeshima, and Koichi Hirata were involved in the study design. Dena H. Jaffe was an investigator in the study and was involved in data collection and statistical analysis. Yongin Kim and Anthony J. Zagar also conducted the statistical analysis.

Prior Presentation. This manuscript includes some data that were previously presented at the 49th Annual Meeting of the Japanese Headache Society, Shizuoka, Japan (November 19-21, 2021).

Disclosures. Yasuhiko Matsumori received personal consultancy fees from Amgen Astellas BioPharma K.K., Daiichi Sankyo Company, Limited, Eli Lilly Japan K.K., and Otsuka
Pharmaceutical Co., Ltd. during the conduct of the study. Kaname Ueda and Mika Komori are employees of Eli Lilly Japan K.K. and own minor shares in Eli Lilly and Company. Anthony J. Zagar and Yongin Kim are employees of, and own minor shares in, Eli Lilly and Company. Dena H. Jaffe is an employee of Cerner Enviza (formerly Kantar Health); Cerner Enviza received research funding for this study from Eli Lilly and Company. Takao Takeshima received personal fees from AbbVie GK, Amgen Astellas BioPharma K.K., Asahi Kasei Pharma Corporation, Bayer Yakuhin, Ltd, Daiichi Sankyo Company, Limited, Eli Lilly Japan K.K., Fujifilm Toyama Chemical Co., Ltd., Janssen Pharmaceutical K.K., Kowa Company, Ltd., Kyowa Kirin Co., Ltd., Lundbeck Japan K.K., Mitsubishi Tanabe Pharma Corporation, Ono Pharmaceutical Co., Ltd, Otsuka Pharmaceutical Co., Ltd., Pfizer Japan Inc., Sawai Pharmaceutical Co., Ltd., Sumitomo Dainippon Pharma Co. Ltd., Novartis Pharma K.K., and UCB Japan Co. Ltd. during the conduct of the study, and personal fees from Takeda Pharmaceutical Company Limited outside the submitted work. Koichi Hirata received research funding from the Japanese Ministry for Health, Labour and Welfare and the Japan Agency for Medical Research and Development and has received personal fees from Amgen Astellas BioPharma K.K., Daiichi Sankyo Company, Limited, Eisai Co., Ltd., Eli Lilly Japan K.K., MSD Co., Ltd., Otsuka Pharmaceutical Co., Ltd., and Pfizer Japan Inc.

Compliance with Ethics Guidelines. Ethical approval for the study was granted by the Research Institute of Healthcare Data Science Ethical Review Board (ID: RI2020003). All survey respondents provided informed consent and all data were anonymized before analysis.

Data Availability. The datasets generated during and/or analyzed during the current study are available from the corresponding author on reasonable request.

Open Access. This article is licensed under a Creative Commons Attribution-NonCommercial 4.0 International License, which permits any non-commercial use, sharing, adaptation, 
distribution and reproduction in any medium or format, as long as you give appropriate credit to the original author(s) and the source, provide a link to the Creative Commons licence, and indicate if changes were made. The images or other third party material in this article are included in the article's Creative Commons licence, unless indicated otherwise in a credit line to the material. If material is not included in the article's Creative Commons licence and your intended use is not permitted by statutory regulation or exceeds the permitted use, you will need to obtain permission directly from the copyright holder. To view a copy of this licence, visit http://creativecommons.org/licenses/by$\mathrm{nc} / 4.0 /$.

\section{REFERENCES}

1. Steiner TJ, Stovner LJ, Jensen R, Uluduz D, Katsarava Z. on behalf of Lifting The Burden: the Global Campaign against Headache. Migraine remains second among the world's causes of disability, and first among young women: findings from GBD2019. J Headache Pain. 2020;21(1):137.

2. Lipton RB, Bigal ME, Kolodner $\mathrm{K}$, Stewart WF, Liberman JN, Steiner TJ. The family impact of migraine: population-based studies in the USA and UK. Cephalalgia. 2003;23(6):429-40.

3. Blumenfeld AM, Varon SF, Wilcox TK, et al. Disability, HRQoL and resource use among chronic and episodic migraineurs: results from the International Burden of Migraine Study (IBMS). Cephalalgia. 2011;31(3):301-15.

4. Steiner TJ, Jensen R, Katsarava Z, et al. Structured Q1 headache services as the solution to the illhealth burden of headache: 1. Rationale and description. J Headache Pain. 2021;22(1):78.

5. Sakai F, Igarashi H. Prevalence of migraine in Japan: a nationwide survey. Cephalalgia. 1997;17(1): $15-22$.

6. Takeshima T, Ishizaki K, Fukuhara Y, et al. Population-based door-to-door survey of migraine in Japan: the Daisen study. Headache. 2004;44(1): 8-19.

7. Hirata K, Ueda K, Komori M, et al. Comprehensive population-based survey of migraine in Japan: results of the ObserVational survey of the Epidemiology, tReatment, and Care Of MigrainE
(OVERCOME [Japan]) study. Curr Med Res Opin. 2021;37(11):1945-55.

8. Takeshima T, Wan Q, Zhang Y, et al. Prevalence, burden, and clinical management of migraine in China, Japan, and South Korea: a comprehensive review of the literature. J Headache Pain. 2019;20(1):111.

9. Shimizu T, Sakai F, Miyake H, et al. Disability, quality of life, productivity impairment and employer costs of migraine in the workplace. J Headache Pain. 2021;22(1):29.

10. Tinelli M, Leonardi M, Paemeleire K, Mitsikostas D, de la Torre ER, Steiner TJ. Structured headache services as the solution to the ill-health burden of headache. 2. Modelling effectiveness and cost-effectiveness of implementation in Europe: methodology. J Headache Pain. 2021;22(1):99.

11. Tinelli M, Leonardi M, Paemeleire K, et al. Structured headache services as the solution to the illhealth burden of headache. 3. Modelling effectiveness and cost-effectiveness of implementation in Europe: findings and conclusions. J Headache Pain. 2021;22(1):90.

12. Igarashi $H$, Ueda $K$, Jung S, Cai Z, Chen Y, Nakamura T. Social burden of people with the migraine diagnosis in Japan: evidence from a populationbased cross-sectional survey. BMJ Open. 2020;10(11):e038987.

13. Kikui S, Chen $\mathrm{Y}$, Todaka $\mathrm{H}$, Asao $\mathrm{K}$, Adachi $\mathrm{K}$, Takeshima T. Burden of migraine among Japanese patients: a cross-sectional National Health and Wellness Survey. J Headache Pain. 2020;21(1):110.

14. Lipton RB, Araujo AB, Nicholson RA, et al. Patterns of diagnosis, consultation, and treatment of migraine in the US: results of the OVERCOME study. Headache. 2019;59(S1):2-3.

15. Headache Classification Committee of the International Headache Society (IHS). The International Classification of Headache Disorders, 3rd edn. Cephalalgia. 2018;38(1):1-211.

16. Lipton RB, Stewart WF, Diamond S, Diamond ML, Reed M. Prevalence and burden of migraine in the United States: data from the American Migraine Study II. Headache. 2001;41(7):646-57.

17. Silberstein S, Loder E, Diamond S, Reed ML, Bigal $\mathrm{ME}$, Lipton RB. Probable migraine in the United States: results of the American Migraine Prevalence and Prevention (AMPP) study. Cephalalgia. 2007;27(3):220-9.

18. Stewart WF, Lipton RB, Dowson AJ, Sawyer J. Development and testing of the Migraine Disability 
Assessment (MIDAS) Questionnaire to assess headache-related disability. Neurology. 2001;56(6 Suppl 1):S20-8.

19. Stewart WF, Lipton RB, Kolodner K, Liberman J, Sawyer J. Reliability of the migraine disability assessment score in a population-based sample of headache sufferers. Cephalalgia. 1999;19(2): 107-14.

20. Iigaya M, Sakai F, Kolodner KB, Lipton RB, Stewart WF. Reliability and validity of the Japanese Migraine Disability Assessment (MIDAS) Questionnaire. Headache. 2003;43(4):343-52.

21. Martin BC, Pathak DS, Sharfman MI, et al. Validity and reliability of the migraine-specific quality of life questionnaire (MSQ Version 2.1). Headache. 2000;40(3):204-15.

22. Jhingran P, Davis SM, LaVange LM, Miller DW, Helms RW. MSQ: Migraine-Specific Quality-of-Life Questionnaire. Further investigation of the factor structure. Pharmacoeconomics. 1998;13(6):707-17.

23. Cole JC, Lin P, Rupnow MFT. Minimal important differences in the Migraine-Specific Quality of Life Questionnaire (MSQ) version. Cephalalgia. 2009;29(11):1180-7.

24. Ohbu S, Igarashi H, Okayasu H, et al. Development and testing of the Japanese version of the MigraineSpecific Quality of Life instrument. Qual Life Res. 2004;13(8):1489-93.

25. Sakai F, Fukuuchi Y, Iwata M, et al. Reliability and validity of the Japanese version of the migraine quality of life survey [in Japanese]. Neurol Therapeutics [Japan]. 2004;21:449-58.

26. Buse DC, Bigal MB, Rupnow M, Reed M, Serrano D, Lipton R. Development and validation of the Migraine Interictal Burden Scale (MIBS): a self-administered instrument for measuring the burden of migraine between attacks. Neurology. 2007;68:A89.

27. Buse DC, Rupnow MFT, Lipton RB. Assessing and managing all aspects of migraine: migraine attacks, migraine-related functional impairment, common comorbidities, and quality of life. Mayo Clin Proc. 2009;84(5):422-35.

28. Reilly MC, Zbrozek AS, Dukes EM. The validity and reproducibility of a work productivity and activity impairment instrument. Pharmacoeconomics. 1993;4(5):353-65.

29. Ueda K, Ye W, Lombard L, et al. Real-world treatment patterns and patient-reported outcomes in episodic and chronic migraine in Japan: analysis of data from the Adelphi migraine disease specific programme. J Headache Pain. 2019;20(1):68.

30. Sakai F, Ozeki A, Skljarevski V. Efficacy and safety of galcanezumab for prevention of migraine headache in Japanese patients with episodic migraine: a phase 2 randomized controlled clinical trial. Cephalalgia Rep. 2020;3:1-10.

31. Shibata M, Nakamura T, Ozeki A, Ueda K, Nichols RM. Migraine-Specific Quality-of-Life Questionnaire (MSQ) version 2.1 score improvement in Japanese patients with episodic migraine by galcanezumab treatment: Japan Phase 2 study. J Pain Res. 2020;13:3531-8.

32. Dodick DW, Ashina M, Sakai F, et al. Vancouver Declaration II on Global Headache Patient Advocacy 2019. Cephalalgia. 2020;40(10):1017-25.

33. Schaetz L, Rimner T, Pathak P, Fang J, Chandrasekhar D, Mueller J. Impact of an employerprovided migraine coaching program on burden and patient engagement: results from interim analysis [abstract]. Cephalalgia. 2019;39(Suppl 1): 384-5.

34. Gallup. Wellcome Global Monitor-First Wave Findings. 2019. https://wellcome.org/reports/ wellcome-global-monitor/2018. Accessed 26 Jun 2021.

35. Nakamura M, Toyama Y, Nishiwaki Y, Ushida T. Prevalence and characteristics of chronic musculoskeletal pain in Japan: a second survey of people with or without chronic pain. J Orthop Sci. 2014;19(2):339-50.

36. Meyers JL, Davis KL, Lenz RA, Sakai F, Xue F. Treatment patterns and characteristics of patients with migraine in Japan: a retrospective analysis of health insurance claims data. Cephalalgia. 2019;39(12):1518-34.

37. Lipton RB, Bigal ME, Diamond M, Freitag F, Reed ML, Stewart WF. Migraine prevalence, disease burden, and the need for preventive therapy. Neurology. 2007;68(5):343-9. 